\title{
Inductive Formulae for General Sum Operations
}

\author{
By Daniel Minoli
}

\begin{abstract}
In this note we report some computer generated formulae for the sum of powers of numbers with nonunitary increments; these reduce to the well-known cases when the increment is one.
\end{abstract}

1. Introduction. Inductive formulae for sums of powers of consecutive integers are well known; the left side of Table 1, based on [1], depicts such formulae for powers up to 10. These are usually derived directly from the fundamental theorem of sum calculus, or via the Bernoulli polynomials as

$$
\sum_{i=0}^{m-1}(1+i)^{n}=\frac{1}{n+1}\left[B_{n+1}(m+1)-B_{n+1}\right] .
$$

Formulae for the case where the increment is not one, cannot apparently be found explicitly in the literature; [4]-[10]. Conceptually these formulae are simple to obtain; however, the algebraic manipulations required tend to be overwhelming. In this note we present the first ten formulae, as obtained on a computer by formal string manipulations.

2. Approach. Let

$$
S^{n}(1, d, m)=\sum_{i=0}^{q}(1+i d)^{n}
$$

where $q=(m-1) / d$ is an integer, $d>0$. We desire a formal closed-form expression for $S^{n}(1, d, m)$. Clearly

$$
\begin{aligned}
S^{n}(1, d, m) & =1+\sum_{k=0}^{n}\left(\begin{array}{l}
n \\
k
\end{array}\right) d^{n-k}+\sum_{k=0}^{n}\left(\begin{array}{l}
n \\
k
\end{array}\right)(2 d)^{n-k}+\cdots+\sum_{k=0}^{n}\left(\begin{array}{l}
n \\
k
\end{array}\right)(q d)^{n-k} \\
& =\frac{m-1+d}{d}+\sum_{k=0}^{n-1}\left(\begin{array}{l}
n \\
k
\end{array}\right)\left[d^{n-k}+2^{n-k} d^{n-k}+\cdots+q^{n-k} d^{n-k}\right] \\
& =\frac{m-1+d}{d}+\sum_{k=0}^{n-1}\left(\begin{array}{l}
n \\
k
\end{array}\right) d^{n-k} \sum_{j=1}^{q} j^{n-k} .
\end{aligned}
$$

The individual terms in the second expression are indeed the entries of the left side of Table 1. The only remaining task is obtaining a formal expression for the first summation, by collecting appropriate terms; this is a rather long and tedious task, particularly for high values of $n$.

Received December 11, 1978.

AMS (MOS) subject classifications (1970). Primary 40-04; Secondary 65B15. 


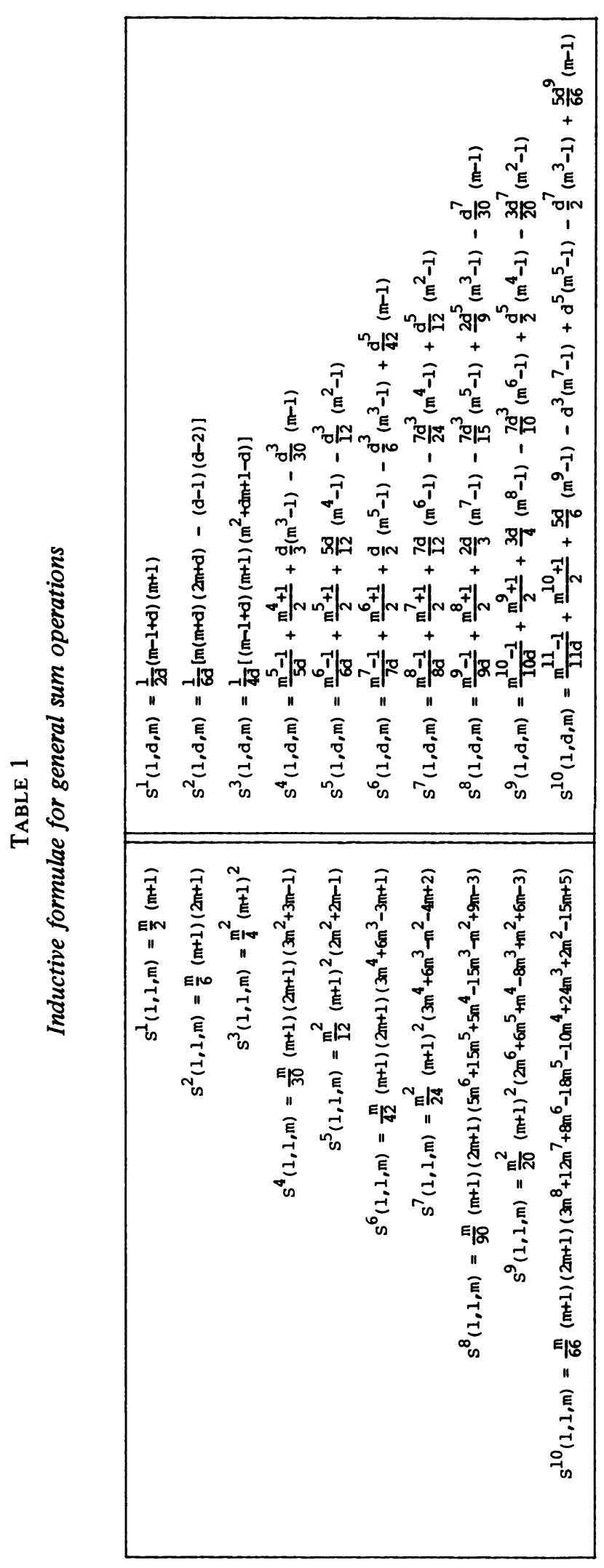


The algebraic manipulations have been carried out by a computer program. CPU time on a dedicated DEC PDP 11/70 was 2 hours; the code consisted of about 400 statements. The results are depicted on the right-hand side of Table 1 . We now have closed-form expressions for summations such as $\Sigma_{j}(1+j \sqrt{A})^{2}$ or $\Sigma_{j}(1+j \pi)^{3}$.

\section{Related Facts.}

Fact 1. Besides brute force computation, the results of Table 1 may be proved by induction on $q$, for a fixed $d$, and $n$.

The following facts can also be proved.

Fact 2. For all $n, d$,

$$
S^{n}(1, d, m) \approx \frac{m^{n+1}-1}{(n+1) d}+\frac{m^{n}+1}{2}+\frac{n d}{12}\left(m^{n-1}-1\right),
$$

which is exact for $n=1$.

Fact 3 . For sufficiently small $d, f(x)$ Riemann integrable, $m \equiv 1 \bmod d$, and

$$
\Lambda^{f}(1, d, m)=\sum_{i=0}^{(m-1) / d} f(1+i d),
$$

there exists a $\delta$ such that

$$
\left|\Lambda^{f}(1, d, m)-\frac{1}{d} \int_{1}^{m} f(x) d x\right|<\delta
$$

In particular, if $f(x)=x^{n}$

$$
\left|S^{n}(1, d, m)-\frac{m^{n+1}-1}{(n+1) d}\right|<\delta .
$$

This is related to the Euler-Maclaurin sum formula [2], and a result on the generalized factorial, [3].

Fact 4. The sum of the odd integers up to $m$ is equal to the sum of the cubes of all integers up to $m$, divided by $m^{2}$; namely, $S^{3}(1,1, m) / m^{2}=S^{1}(1,2, m)$.

ITT Domestic Transmission Systems, Inc.

2 Broadway

New York, New York 10004

1. CRC Handbook of Tables for Probability and Statistics (W. H. Beyer, Ed.), The Chemical Rubber Co., Cleveland, Ohio, 1966.

2. M. ABRAMOWITZ \& I. STEGUN, Handbook of Mathematical Functions, Dover, New York, 1964.

3. D. MINOLI, "Asymptotic form for the generalized factorial," Rev. Colombiana Mat., v. $11,1977$.

4. K. S. MILLER, An Introduction to the Calculus of Finite Differences and Difference Equations, Holt, New York, 1960.

5. C. JORDAN, Calculus of Finite Differences, 3rd ed., Chelsea, New York, 1965.

6. F. B. HILDEBRAND, Finite-Difference Equations and Simulations, Prentice-Hall, Englewood Cliffs, N. J., 1968.

7. G. BOOLE, Calculus of Finite Differences, 4th ed., Chelsea, New York, 1958.

8. I. S. GRADSHTEYN \& I. M. RYZHIK, Table of Integrals, Series, and Products, 4th ed., Academic Press, New York, 1965.

9. ELDON R. HANSEN, A Table of Series and Products, Prentice-Hall, Englewood Cliffs, N. J., 1975.

10. I. J. SCHWATT, An Introduction to the Operations with Series, Univ. of Pennsylvania Press, 1924; reprinted by Chelsea, New York, 1962. 